http://jmscr.igmpublication.org/home/ ISSN (e)-2347-176x ISSN (p) 2455-0450 crossref DOI: https://dx.doi.org/10.18535/jmscr/v8i9.18

\title{
Presurgical Nasoalveolar Molding Therapy in Bilateral Cleft Lip and Palate - A Case Report
}

\author{
Authors \\ Jaziya.$Z^{1}$, Rita Zarina. $A^{2}$, Digesh Balachandran ${ }^{3}$, Fathima.$S^{4}$ \\ ${ }^{1}$ Junior Resident, Dept. of Pediatric and Preventive Dentistry, Government Dental College, \\ Thiruvananthapuram, Kerala, India \\ ${ }^{2}$ Professor and Head, Dept. of Pediatric and Preventive Dentistry, Government Dental College, \\ Thiruvananthapuram, Kerala, India \\ ${ }^{3}$ Associate Professor, Dept. of Pediatric and Preventive Dentistry, Government Dental College, \\ Thiruvananthapuram, Kerala, India \\ ${ }^{4}$ Junior Resident, Dept. of Pediatric and Preventive Dentistry, Government Dental College, \\ Thiruvananthapuram, Kerala, India
}

\begin{abstract}
Cleft lip and palate cases require presurgical orthopaedics to achieve the normal alignment of the cleft maxillary segments prior to primary cheiloplasty. Management of the protruding/deviated premaxilla in patients with complete bilateral cleft lip and palate is a challenging problem. Various passive and active methods have been developed for the presurgical orthopaedic treatment. However, most of these treatments are complicated and laborious for the patients, parents and clinicians. This article aims to review the preferable options for the management of bilateral cleft lip and palate in children and to discuss a case report where an active intraoral appliance comprising of two components, that is the acrylic premaxillary and palatine process plates, connected with two elastic chains had been successfully employed and satisfactory results obtained.
\end{abstract}

Keywords: Cleft lip and palate; Presurgical orthopaedics; Nasoalveolar molding.

\section{Introduction}

Cleft lip and palate is one of the most frequent congenital malformations that affect the maxillofacial complex. Such anomalies can have several consequences such as impaired suckling, defective speech, deafness, malocclusion, gross facial deformity and serious psychological issues and bring tremendous stress on the entire family. ${ }^{[1]}$ Of all congenital malformations, it ranks third and has a multifactorial etiology. ${ }^{[2]}$
Current guidelines promote the use of presurgical orthopaedics from birth, to align the segments of the cleft maxilla and nasal molding in the first few months, prior to the surgical reconstruction of the lip and palate. This procedure is based on research undertaken by Matsuo and Hirose. ${ }^{[3]}$ They report that the nasal cartilage is still in the developmental stage in newborns and is subject to relocation due to cartilage plasticity, which has high levels of hyaluronic acid that circulates in the body several weeks after birth. ${ }^{[4]}$ 
Grayson and Cutting ${ }^{[5]}$ were among the first to combine the concepts of presurgical orthopaedics and preoperative treatment of cleft lip nose deformity. They developed the concept of nasoalveolar molding (NAM), which combined a nasal molding stent with a passive preoperative molding apparatus in the treatment of patients with cleft palate. A study conducted by Spengler et al. ${ }^{[6]}$ showed that presurgical therapy has major advantages for the treatment of bilateral cleft lip and palate.

Presurgical orthopaedics is indicated for infants with a large cleft lip and palate defect and a severe maxillary skeletal malformation. Its main aim is to achieve a normal anatomy at an early stage by reducing the opening size and re-establishing the correct anatomic ratio of the maxillary cleft segments. ${ }^{[7]}$ It also helps to direct the growth of the segments where the maxilla is divided, enhance lingual function, promote newborn feeding, mold the nasal cartilages and reshape the columella. ${ }^{[8]}$

The Presurgical orthopaedic treatment is completed approximately by 5 months of age, before the primary palate is closed, after which the device is removed and the first surgery is performed. ${ }^{[9]}$ In cheiloplasty with protruding/deviated premaxilla in complete bilateral cleft lip and palate, it is difficult to symmetrically approximate the skin, mucosa and muscle with low tension, resulting in postoperative functional and cosmetic problems. ${ }^{[10]}$ Therefore, various methods including active/passive and intraoral/extraoral appliances have been described for the presurgical orthopaedic treatment of complete bilateral cleft lip and palate. These methods or appliances may vary from cases to case, depending on the severity of the condition.

\section{Case Report}

A 10-day-old male newborn, diagnosed with complete bilateral cleft lip and palate, Veau's classification Group 4, received care at the Sree Avittam Thirunal Hospital and Government Dental College Thiruvananthapuram by a multidisciplinary team of health specialists that included, in the first phase; a pediatric surgeon, an obstetrician, a kinesiologist, a speech therapist, a pediatric dentist and a psychologist. General examination showed all parameters to be within normal limit, except for the orofacial cleft. On detailed examination a good jaw ratio, complete cleft of the palate with the lateral segments displaced by $15-18 \mathrm{mms}$, premaxilla displaced about $6 \mathrm{mms}$ from the lateral segments and with greater displacement to the right.

The treatment of the newborn was coordinated by a multidisciplinary team to achieve complete rehabilitation and to improve the infant's living conditions; feeding, phonation and aesthetics. Presurgical orthopaedic rehabilitation was started at an early stage with lip taping and the insertion of a removable active intra oral plate which was in place until the infant was 5 months old.

Majority of care givers complained about skin irritation when topoplast alone was used for extraoral lip taping. Therefore we insisted on the use of micropore skin adhesion tape prior to topoplast application. This greatly enhanced patient compliance. Hence it is advocated that prior to topoplast application the use of micropore as a protective mechanism will promote faster closure of the segments.

The intraoral active appliance comprised of two acrylic components, an anterior premaxillary plate (PM plate) fitting the surface of the premaxilla and a split palatine process plate (PP plate) with an activated/expanded jack screw attached to the midline, fitting the palatine processes. PM and PP plate were connected with two elastic chains.

The appliance was inserted at the age of 3 months. It was stabilized intraorally with the help of waxed dental floss. The dental floss penetrated the PM plate through two holes and it was adapted with lip taping. The jack screw was fabricated on a separate acrylic plate which was then activated and attached to the PP plate. The expanded jack screw of the PP plate was deactivated and elastic chains connected in between the PM and PP plates were activated on regular intervals.

Measurements were taken on cast models to compare before and after treatment results. They showed significant reductions in protrusion and 


\section{JMSCR Vol||08||Issue||09||Page 87-91||September}

deviation of the premaxilla, and in the width of the defect, which was observed in subsequent checkups. After a 2-month consolidation period, at the age of 5 months, Mulliken's bilateral cheiloplasty was performed.

Good results were obtained and there was no evidence of lip retraction. According to the care protocol, a kinesiology treatment was applied to heal the wound using mesotherapy and ultrasound, among other techniques. Both the appearance and the texture of the skin improved, with greater mobility and more action capacity and functionality of perioral muscles. A palatoplasty procedure is planned to be performed as the infant turns a year old. In the dental checkup after the cheiloplasty, the infant's mother was given information about long term dental rehabilitation needs, as well as a healthy diet. The infant will be kept under regular dental review till palatoplasty, to check for any relapse.

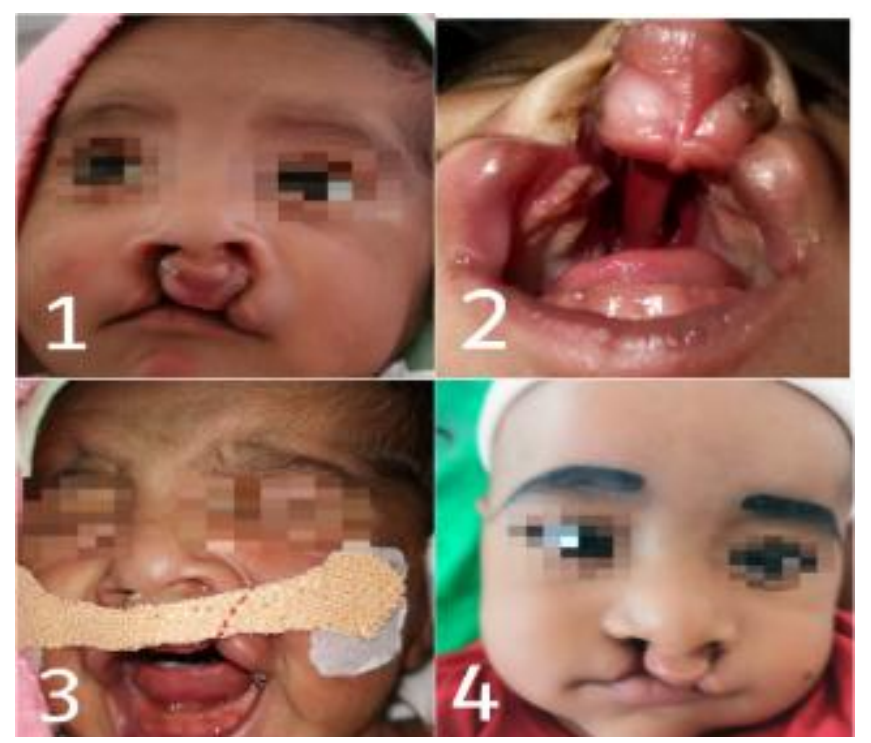

Figure 1: Pretreatment extraoral (1) and intraoral (2) photos. Extraoral lip taping (3) and the result, 2 months after therapy (4).

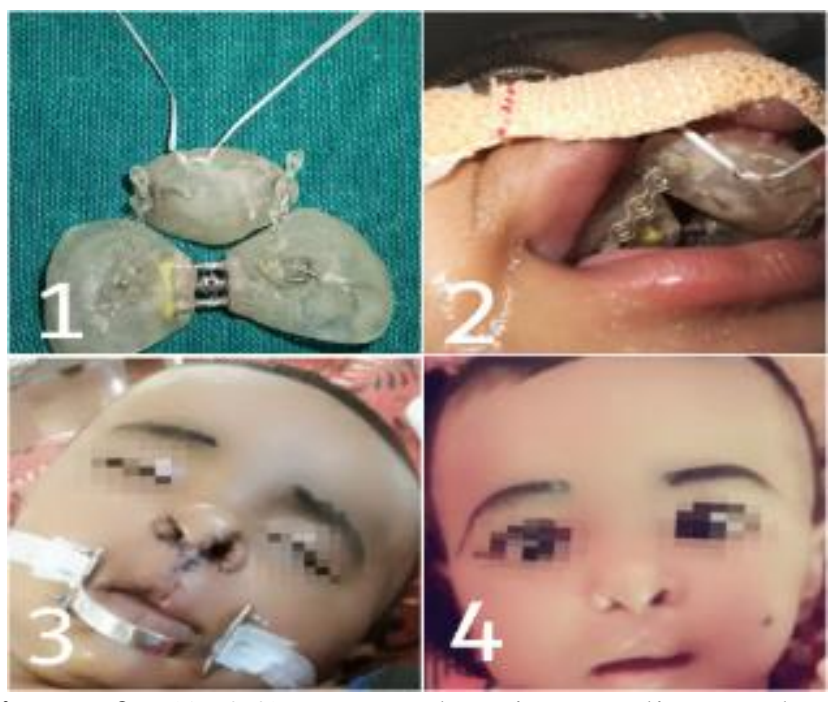

Figure 2: (1 \&2) Intraoral active appliance along with lip taping. (3 \&4) Postoperative views just after and at 1 month after cheiloplasty.

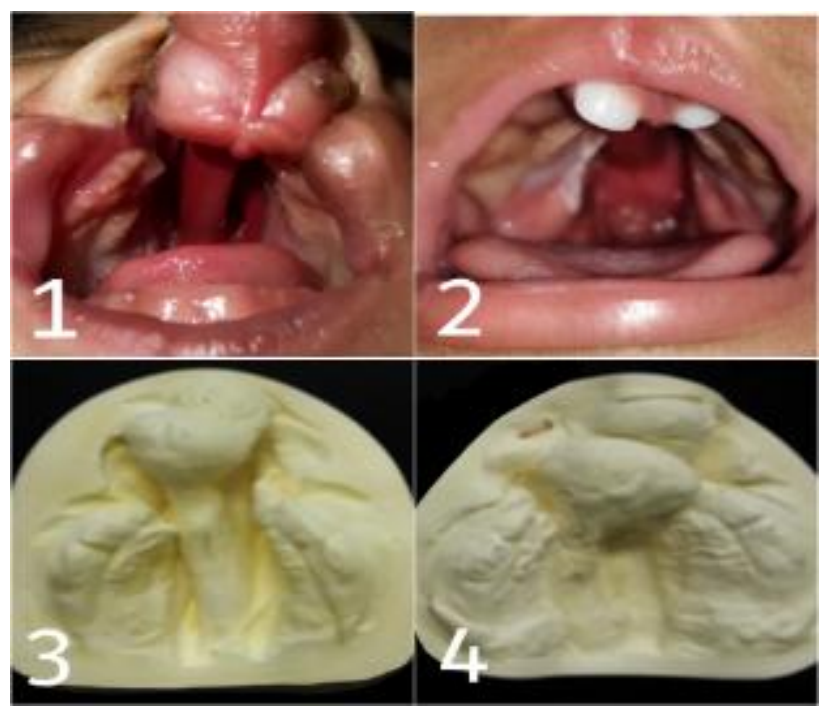

Figure 3: Pre-treatment (chelioplasty) and posttreatment ( $1 \& 2)$ occlusal views. Maxillary cast obtained at pretreatment (left) and post treatment (right) $(3 \& 4)$

\section{Discussion}

Presurgical orthopaedics allows for an appropriate alignment of alveoli, lip and nose, which results in better surgical outcomes. The size of the cleft palate is significantly reduced before the surgery, which allows the surgeon to successfully perform a gingivoperiosteoplasty and facilitates closure of the nasolabial gap by reducing the tension in soft tissues. Once the alveolar segments are better positioned and bone bridges through the defect become more solid, permanent teeth have a greater probability of 
erupting in a good position with the necessary periodontal support. ${ }^{[11]}$

Nasoalveolar molding also results in fewer and less complicated surgeries to restore and maintain acceptable nasolabial aesthetics such as the growth of the nose. ${ }^{[12]}$ Another advantage was shown by Lee et $\mathrm{al}^{[13]}$, who reported that midfacial growth on the sagittal and vertical planes are unaffected. Besides, it results in good long-term nasal aesthetics, corrects the wrong position of the nasal cartilages and the alar base on the affected side, extends the columella, and restores the normal contour of the maxillary arch. It also helps in complete separation of the oral and nasal cavities, thus minimizing the entry of food into the nasal cavity, reducing adverse effects on the nasal mucosa and facilitating feeding. ${ }^{[7]}$ It eliminates or reduces the future need for a nasoalveolar bone graft. $^{[14]}$ It improves breathing and the development of orofacial functions; deglutition, phonation and feeding. ${ }^{[15]}$ It corrects the long-term disposition of facial structures and also has psychological and social benefits for parents and patients. ${ }^{[16]}$

In a prospective study conducted by Spengler ${ }^{[17]}$ in Houston, in patients with bilateral cleft lip and palate, measurements were taken in cast models to compare before and after treatment results. They showed significant reductions in protrusion and deviation of the premaxilla, and in the width of the defect, which was observed in subsequent checkups. During the treatment, patients attended periodical checkups with the pediatric dentist, as was the case of this patient, to prevent and monitor dental problems, as well as otolaryngologic and velopharyngeal evaluations at least once a year.

A unique characteristic of our appliance is the pressure exerted by the PM plate on the anterior surface of the premaxilla rather than on the prolabial skin. This makes our technique less invasive on the prolabial skin. Moreover, the premaxillary vermilion mucosa was expanded by the PM plate and its traction force. Accordingly, during cheiloplasty, the premaxillary mucosa could be utilised not only for the vestibule but also for reinforcing the nasal floor.
This novel appliance is simple, lightweight and cost-effective, as compared to other available modalities and can be inserted without general anesthesia. There was no history of detachment of the appliance or its components during the treatment. We assume that traction by the elastic chains and the extraoral connection to the taping unit greatly enhanced the retention of the appliance. However, further follow-up and investigations are needed to determine the long-term outcomes of treatment using this novel appliance, including its effects on maxillary growth and tooth formation.

\section{References}

1. Kaul R, Jain P, Saha S, Sarkar S. Cleft lip and cleft palate: Role of a pediatric dentist in its management. Int $\mathbf{J}$ Pedod Rehabil 2017;2:1-6.

2. Rivera B, Tobar M, Chianale T, Monti F, Flores P. Presurgical orthopedics in children with cleft palate. Case Report. :6.

3. Matsuo K, Hirose T. Nonsurgical correction of cleft lip nasal deformity in the early neonate. Ann Acad Med Singapore. 1988 Jul;17(3):358-65.

4. Grayson BH, Shetye PR. Presurgical nasoalveolar moulding treatment in cleft lip and palate patients. Indian J Plast Surg. 2009 Oct; 42:56-61.

5. Grayson BH, Cutting CB. Presurgical nasoalveolar orthopedic molding in primary correction of the nose, lip, and alveolus of infants born with unilateral and bilateral clefts. Cleft Palate Craniofac J 2001;38:193- 8 .

6. Chavarria C, Gateno J, Spengler A, Teichgraeber J, Xia J. Presurgical nasoalveolar molding therapy for the treatment of bilateral cleft lip and palate: A preliminary study. Cleft Palate Craniofac J. 2006 May; 43(3):321-8.

7. Carrasco L, Faraggi M, Merino A. Rinoseptoplastía en pacientes fisurados. Rev Otorrinolaringol Cir Cabeza Cuello. 2011; 71(2):171-178. 
8. Delgado-Muñoz M, Herrero E, RomanceGarcía A, Romero-Maroto M. Tratamiento ortopédico prequirúrgico de la fisura palatina con la filosofía de Latham. RCOE. 2005; 10(2):199-204.

9. Broder H, Chan J, Sischo L, Smith C, Stein M, Van Aalst J. Nasoalveolar Molding: Prevalence of Cleft Centers Offering NAM and Who Seeks It. Cleft Palate Craniofac J. 2012 May;49(3):270-275.

10. Bitter K. Latham's appliance for presurgical repositioning of the protruded premaxilla in bilateral cleft lip and palate. J Craniomaxillofac Surg 1992;20:99e110.

11. Bravo Rivera L, Muñoz Tobar D, Torres Chianale F, Fierro Monti C, Pérez Flores A. Ortopedia prequirúrgica en niños fisurados: Reporte de caso clínico. Odontoestomatología. 2015 May;17(25):539.

12. Maillard S, Retrouvey J-M, Ahmed MK, Taub PJ. Correlation between Nasoalveolar Molding and Surgical, Aesthetic, Functional and Socioeconomic Outcomes Following Primary Repair Surgery: a Systematic Review. J Oral Maxillofac Res [Internet]. 2017 Sep 30

13. Lee CTH, Garfinkle JS, Warren SM, Brecht LE, Cutting CB, Grayson BH. Nasoalveolar molding improves appearance of children with bilateral cleft lip-cleft palate. Plast Reconstr Surg. 2008 Oct;122(4):1131-7.

14. Grabowski R, Gundlach K, Kopp H, Stahl F. Presurgical orthopaedic treatment of newborns with clefts - functional treatment with long-term effects. J Craniomaxillofac Surg. 2006; 34(2):34-44.

15. Kinouchi N, Horiuchi S, Yasue A, Kuroda Y, Kawai N, Watanabe K, et al. Effectiveness of presurgical nasoalveolar molding therapy on unilateral cleft lip nasal deformity. Saudi Med J. 2018 Feb;39(2):169-78.

16. Adali N, Mars M, Noar J, Petrie A, Sommerlad B. Presurgical Orthopedics Has No Effect on Archform in Unilateral Cleft
Lip and Palate. Cleft Palate Craniofac J. 2012 Jan; 49(1):5-13.

17. Spengler AL, Chavarria C, Teichgraeber JF, Gateno J, Xia JJ. Presurgical nasoalveolar molding therapy for the treatment of bilateral cleft lip and palate: A preliminary study. Cleft Palate-Craniofacial J Off Publ Am Cleft Palate-Craniofacial Assoc. 2006 May;43(3):321-8. 